\title{
Il sistema difensivo costiero della Sardegna meridionale nella cartografia dei secoli XVI-XVII
}

The coastal defensive system of Southern Sardinia in the cartography of the sixteenthseventeenth centuries

\section{Sebastiana Nocco}

Istituto di Storia dell'Europa Mediterranea - Consiglio Nazionale delle Ricerche, Cagliari, Italy, nocco@isem.cnr.it

\begin{abstract}
Starting from the sixteenth century, the coastal landscape of Sardinia suffered deep transformations related to the construction of defensive structures to protect the inhabitants of the towns and land resources. Coastal towers and fortresses are the most evident signs of these interventions. This paper aims to reconstruct these processes through archival and cartographic documents produced between the second half of the sixteenth century and the first decades of the seventeenth century. Particularly interesting is the comparison between the maps depicting the coastal area between Cagliari and Quartu and its hinterland drawn in 1577 by Rocco Capellino and those drawn underneath the viceroy Vivas in 1623-1625. Cartographic and archive documents can reveal interesting data to reconstruct the changes that occurred in this period, as a result of the interventions aimed at the defense of the coast and the towns with the construction of the first watchtowers and the progressive modernization of the outer walls of the fortress of Cagliari and its ramparts' raising.
\end{abstract}

Keywords: Sardinia, Modern age, historical cartography, defensive system, Cagliari.

\section{Introduzione}

Le isole hanno sempre avuto un ruolo strategico nel controllo dello spazio mediterraneo, in tempo pace e di guerra. Le differenti percezioni degli ambienti costieri nel corso dei secoli hanno stimolato la produzione di cartografie dalle valenze e significati diversi, condizionate dal contesto culturale e sociale, dalla committenza, dalle tipologie d'uso cui erano destinate. In ogni epoca, infatti, le carte sono state utilizzate per governare e viaggiare, far la guerra e i commerci o per puro godimento estetico.

Con la sua forma chiusa, l'isola si presenta come l'oggetto cartografico per eccellenza, le cui coste possono essere rilevate e disegnate interamente

dal mare. Il perimetro costiero rappresenta altresì un confine naturale, fisso e stabile, non suscettibile di espansione in quanto delimitato dal mare ma, al contempo, fascia permeabile nella quale "lo spazio del confine si configura, di volta in volta, come luogo dello scambio, del pericolo, dell'interesse, della precarietà, del conflitto, della difesa, della marginalità, dei diritti contesi, che il "racconto" cartografico riesce a svelare per spaccati sia sincronici che diacronici e ricomporre nella sinottica incisività della rappresentazione" (Dai Prà, 2010, p. 168).

La Sardegna, isola al centro del Mediterraneo occidentale, per tutto il Basso Medioevo aveva 
costituito un importante crocevia per le potenze interessate al controllo dei traffici marittimi mercantili, tappa fondamentale delle rotte mediterranee, in particolare di quella ruta de las islas che dalle Baleari conduceva verso gli scali commerciali d'Oriente. Carte nautiche e portolani medioevali testimoniano questo ruolo strategico e restituiscono un profilo costiero delineato con cura, sebbene alla piccola scala, cui si aggiungono informazioni su rotte, porti e approdi, in particolare i numerosi tragitti d'altura che, dipartendosi da capi, promontori e isolette minori della Sardegna, la collegavano con i più importanti scali mediterranei (Quaini, 1974).

Tuttavia, questa stessa centralità sembra perdere ben presto valore se, nei quasi quattrocento anni di storia iberica (1324-1720), "l'isola si caratterizza per la quasi totale assenza di rappresentazioni sia letterarie sia cartografiche [...] riconducibili direttamente o indirettamente al governo centrale prima di Barcellona e poi di Madrid" (Zedda Macció, 2007, pp. 318-319). Peraltro, solo raramente la Sardegna è disegnata dai Sardi; molto spesso, invece, in particolare nel Cinquecento, ci troviamo di fronte a immagini pubbliche disegnate da lontano per completare $\mathrm{i}$ più prestigiosi atlanti e non per effettivo interesse. Così, gli unici documenti che attestano un'attività cartografica promossa dagli Spagnoli in Sardegna e caratterizzati da una visione analitico-progettuale del territorio sono i disegni "carto-topografici", ancora oggi conservati negli archivi, legati alla funzione politico-militare attribuita alle coste ed elaborati nel momento in cui l'isola diviene avamposto della cristianità di fronte alla minaccia turca. ${ }^{1}$

\subsection{Progettare la difesa della Sardegna spa- gnola}

Con l'intensificarsi della guerra di corsa e delle scorrerie musulmane, dalla metà del Cinquecento nel Regnum Sardiniae, come negli altri possedimenti mediterranei della Corona di Spagna, furono avviate una serie di attività finalizzate a tutelare le popolazioni dei centri abitati e le risorse del territorio. La Sardegna, infatti, sebbene esclusa dai grandi scontri, registrava un'impressionante sequela di azioni corsare che andavano dalla razzia di uomini e merci sulle coste o sulle imbarcazioni dedite agli scambi commerciali, ai saccheggi di villaggi anche lontani dal mare con la cattura degli abitanti (Mele, 2000).

Le esigenze difensive della monarchia resero indispensabile il lavoro di ingegneri e architetti militari, spesso italiani, impegnati in tutti i territori della Corona per predisporre la difesa delle frontiere marittime e terrestri. Li vediamo viaggiare per informare il sovrano e conoscere il territorio nel quale costruire nuove fortificazioni o adeguare le antiche, "dibuan, trazan, construyen y asesoran", come testimoniano le relazioni e i disegni pervenutici (Cámara, 1998; Zedda Macció, 2008).

I grandi cantieri delle fortificazioni modificarono profondamente l'assetto urbanistico e il paesaggio costiero del Mediterraneo occidentale, dalle forme architettoniche ai materiali usati, sperimentando tecniche costruttive che attenuassero gli impatti dell'artiglieria senza crollare (Cámara, 1998).

Nell'ambito di queste vicende, il presente lavoro intende focalizzare l'attenzione sul caso sardo e in particolare sul processo di fortificazione del settore meridionale dell'isola e della capitale, ripercorso attraverso i documenti prodotti tra la seconda metà del Cinquecento e il primo quarto del Seicento.

Il materiale iconografico, letto e analizzato insieme alla documentazione scritta conservata negli archivi italiani ed europei, costituisce una fonte preziosissima per comprendere le dinamiche che hanno interessato l'area. Infatti, come auspicava Lucio Gambi: "i documenti cartografici potranno ricevere la lettura storica più completa e fedele solo quando verranno raccordati con gli ambiti politici e culturali in cui hanno esercitato la loro funzione di strumento giuridico, urbanistico, agronomico, fiscale, militare, ecc. e verranno posti in relazione con la documentazione di qualunque genere che la classe dirigente ha cumulato per conoscere meglio il territorio" (Gambi, 1987, p. 855).

Nel nostro caso di studio le carte manoscritte, elaborate a seguito delle indagini conoscitive e 
perlustrazioni del territorio, testimoniano alcune fasi della progettazione degli interventi a tutela della capitale del Regnum Sardiniae e del suo hinterland, fino alla predisposizione di un piano complessivo comprendente anche una rete di torri in collegamento visivo fra loro.

Come si evince dalla lettura della documentazione superstite, in particolare dallo scambio di una fitta corrispondenza con la Corte e con il Consiglio Supremo di Aragona -organo costantemente informato su qualsivoglia attività inerente le fortificazioni- un ruolo importante nella promozione e conduzione di queste iniziative è riconducibile spesso ai viceré. Diretti interlocutori del sovrano, si confrontano e spesso si scontrano con le figure istituzionali e professionali coinvolte nei cantieri, in particolare gli ingegneri, come confermano le missive inviate a Madrid da questi ultimi, spesso accompagnate dai disegni esplicativi dei progetti (Zedda Macció, 2007; Mele, 2006, 2017).

Non a caso, nel momento in cui Filippo II istituì l'Amministrazione delle Torri, nel 1587, con il compito di gestire il nuovo sistema difensivo isolano, stabilì che fosse diretta personalmente dal viceré. E, qualche anno prima, nel Parlamento del 1583, era stato proprio il viceré Miguel de Moncada a sottolineare che il Regno di Sardegna, dopo la perdita della Goletta, si trovasse "en frontera de Tunes y Biserta, y de toda la Berberia, y $[\ldots]$ muy peligroso de ser invadido", così da indurlo a rafforzare l'artiglieria, incaricare gli ingegneri militari di ristrutturare le piazzeforti, ma soprattutto ribadire che fosse ormai improrogabile avviare la costruzione delle torri per proteggere la frontiera marittima (Mele, 2000, p. 59).

\section{Il disegno del paesaggio fortificato}

Il settore meridionale della Sardegna, per il fatto stesso di ospitare la capitale del Regno -Castel di Castro, primo nucleo della moderna Cagliari, fondato dai Pisani agli inizi del Duecento- era guardato sempre con grande attenzione dai governanti che impiegarono ingenti risorse per la sua tutela. I catalano-aragonesi ne compresero subito il ruolo strategico di clau de toda la isla, la cui salvaguardia si rivelava, dunque, fonda- mentale per il mantenimento del potere e del regno stesso (Urban, 2000). Una consapevolezza che permase anche nella prima età moderna, allorché l'isola, seguendo le sorti della Corona d'Aragona, divenne parte della Corona di Spagna.

Ai primi del Cinquecento Cagliari e Alghero erano le due città chiave dalla Corona, che vedeva nelle piazzeforti il cardine della strategia difensiva isolana. Così nel 1534 Carlo $\mathrm{V}$ affidava al nuovo viceré de Cardona la guarda y difensión del Reyno, in cui "la Ciudad de Cáller es la cabeça de aquel Reyno y conviene que ste siempre bien proveyda fortificada y bastecida" (Manconi, 2001, pp. 382, 393).

Lo stesso sovrano, dopo qualche tempo, provvide ad affidare il delicato compito a un ingegnere e, dopo il rifiuto del milanese Giovanni Maria Olgiati a causa dell'età avanzata e del precario stato di salute, nel 1552 incaricò Rocco Capelli$\mathrm{no}^{2}$, al quale furono affiancati, nel corso dei vent'anni trascorsi nell'isola, altri ingegneri. Tra le carte a varia scala elaborate dal Capellino al termine del suo operato, vorremmo in questa sede soffermarci sul disegno che occupa i ff. $27 v$ e $28 r$ del codice Barberini e illustra il Golfo di Cagliari da Capo Pula allo stagno di Quartu e il relativo entroterra.

$\mathrm{Al}$ centro della carta figura la città di Cagliari (Fig. 1), inquadrata dal versante occidentale, lungo il quale sono evidenziati il circuito murario e i bastioni già edificati, dal settore di San Pancrazio (la cui torre è ben riconoscibile) a Marina (Alberti, 1970; Principe, 1981).

La piccola veduta prospettica, tuttavia, si limita ad illustrare solo i quartieri dentro il perimetro fortificato -Castello e Marina, la cui trama urbana è appena abbozzata con il profilo delle case e dei campanili- mentre non figurano Villanova e Stampace, secondo un processo comune alle piante militari dell'epoca. Le due appendici non figuravano neppure nella pianta della piazzaforte disegnata dallo stesso Capellino (Codice Barberini, ff. 41v-42r.), in cui Stampace era comunque richiamato dal toponimo sta in pace (Nocco, 2016, 2018). 
Nel gradevole disegno, oltre a un abbozzo dell'orografia e idrografia (in particolare gli stagni), si riconoscono i piccoli disegni prospettici dei centri abitati di Quarto, Selargio, Pavoli, Pi$r i$ (rispettivamente Quartu, Selargius, Monserrato, Pirri), il castello di San Michele, la chiesa di San Bartolomeo, nei pressi del promontorio di Sant'Elia, nella cui estremità viene ubicata la torre omonima. Attigue alla città di Cagliari sono le chiese di Bonoaier (Bonaria) e S. Brancac (San Pancrazio). Nella parte bassa del disegno, in prossimità delle rive dello Stagnio de le pescere (stagno di Santa Gilla, collegato con il mare attraverso il canale della Scafa), compaiono le chiese di S. Pera (San Pietro dei Pescatori) e $S$. Venere (S. Venerius, S. Avendrace), quest'ultima affiancata da un piccolo gruppo di case, nell'area in cui dovette sorgere l'antica capitale del Giudicato di Càlari, Santa Gilla, distrutta nel 1257-1258 dai Pisani. ${ }^{3}$

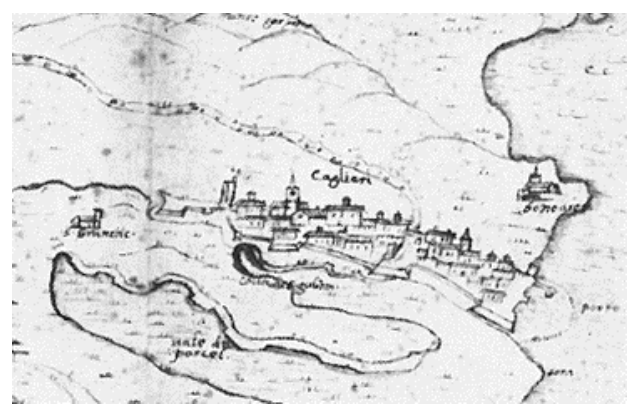

Fig. 1. Rocco Capellino, Cagliari, particolare del disegno delle coste e dintorni di Cagliari, 1577 (riproduzione in Principe, 1981, p. 72).

Nel periodo in cui Capellino visitava il territorio sardo, la vigilanza delle coste era ancora affidata a un servizio di sentinelle e ronde a cavallo a carico delle comunità locali o dei signori feudali ma, proprio in quegli anni, si approntava il progetto di edificazione delle torri costiere, per le quali il capitano Camós, su incarico del viceré, aveva identificato i siti più adatti. La relazione di quest'ultimo documenta, infatti, l'esistenza di numerosi posti di guardia e di quattordici torri già operative nel 1572, tra cui quella di Sant'Elia, fatta edificare dai Pisani nel 1282 e illustrata anche nel disegno di Rocco Capellino (Zedda Macció, 2007).
Le altre torri di epoca moderna erano state invece edificate nella prima metà del secolo su iniziativa delle città regie, di imprenditori o signori feudali a tutela dei propri interessi, come già avveniva per le guardie, che assicuravano protezione ai centri abitati maggiori e alle aree nelle quali erano presenti attività economiche da tutelare, trascurando le aree più povere e a bassa densità demografica (Mele, 2000).

Cagliari, oltre alla sorveglianza all'interno della città, si faceva carico di pagare quattro coppie di sentinelle che operavano a Capo Carbonara, nel Capo di Pula, sul promontorio di Sant'Elia e nella torre di San Pancrazio per vigilare sul golfo e sulle imbarcazioni che entravano in porto, mentre Quartu retribuiva due guardie di stanza nella sommità di Cala sa Reyna (Mele, 2000).

In prossimità del Golfo di Cagliari, tuttavia, il disegno del Camós si fa meno preciso, facendo ipotizzare che proprio per quell'area -di grande interesse strategico-militare in quanto avamposto del sistema di difesa delle frontiere del regno, in cui per giunta si trovava la capitale- potessero sussistere ancora incertezze sui siti in cui impiantare le torri di difesa. È inoltre probabile che il compito assegnato al Camós riguardasse piuttosto il circuito generale dell'isola, dato che la difesa dell'area di Cagliari era, invece, affidata agli ingegneri militari addetti alle fortificazioni delle città regie (Zedda Macció, 2007).

Nel 1574, in seguito alla sconfitta della Goletta, Filippo II era stato costretto a ripensare la strategia difensiva ${ }^{4}$ e in quella circostanza il Camós aveva elaborato una seconda relazione, nella quale sollecitava che si costruissero quanto prima almeno trenta torri nelle coste meridionali e occidentali, da Capo Carbonara a Capo Falcone (Mele, 2000; Zedda Macció, 2007).

Intanto proseguivano le fabbriche nelle aree strategiche: nel 1578 risultano già operative due nuove torri realizzate dalla città di Cagliari a Cala Bernat e Cala Mosca, nei pressi del Capo Sant'Elia, e due finanziate dalla città di Quartu. Per tale ragione quest'ultima, nel Parlamento del 1583 aveva chiesto l'esonero dal sostentamento delle relative guarnigioni (Rassu, 2005). 
In un documento del giugno 1580 il viceré de Moncada informava il sovrano che si stavano completando le dieci torri previste nel Golfo di Cagliari. Queste prime opere non furono comunque sufficienti a impedire che nel 1582 i villaggi di Quartu, Quartucciu, Pirri e Pauli fossero saccheggiati (Mele, 2000); un altro sbarco, tentato nella spiaggia di Quartu nel 1620, fu invece respinto.

Qualche anno più tardi Giovanni Francesco Fara segnalava nella Chorographia Sardiniae le nuove torri dell'isola, tra cui quelle di recente edificate nella costa meridionale che coprivano il tratto da Capo Carbonara a Sarroch, nel tentativo di difendere al meglio le coste intorno alla capitale (Rassu, 2005). Alcune di queste risultavano già in riparazione alla fine del secolo e nei primi anni del successivo, spesso in concomitanza con minacce di incursioni. Così, nel 1615 , la notizia che la flotta ottomana fosse salpata da Costantinopoli con destinazione ignota aveva indotto a ripristinare le guarnigioni di alcune torri del Golfo di Cagliari che erano state in precedenza ridimensionate (Mele, 2000).

La difesa del territorio e delle coste antistanti la capitale restava comunque una preoccupazione costante. Tra il 1623 e il 1625 il viceré Juan Vivas si fece promotore di un piano difensivo per il potenziamento del litorale cagliaritano, compresa la zona di Sant'Elia, di cui ci restano le relazioni e alcuni disegni dei quali, al momento, non è stato individuato l'autore.

All'inizio del Seicento la piana di San Bartolomeo e la montagna di Sant'Elia costituivano l'unica zona di pascolo per il bestiame degli abitanti di Cagliari e di approvvigionamento di legname per i poveri, che nel Parlamento del 1614 chiedevano al sovrano di essere tutelati dai soprusi del governatore (Ortu, 1995).

L'area del Capo Sant'Elia, dotata di una torre di avvistamento fin dal 1282 , vigilata di continuo e munita di recente di altre torri che riuscivano a coprire le varie baie e insenature, era di importanza fondamentale per l'accesso alla città tramite il "puerto de Calamoscas que está poco más de un tiro de culebrina desde Castillo de Cáller" (Mele, 2006).
Per questa ragione il viceré ne fece rilevare una pianta (Fig. 2), inedita fino a pochi anni fa, che venne allegata a una relazione inviata a Corte nel giugno 1623, la Planta d(e) S(an)t Elias che illustra il promontorio di Sant'Elia, la piana di San Bartolomeo, la montagna e la chiesa di Sant'Elia, le saline e le torri già edificate. ${ }^{5}$

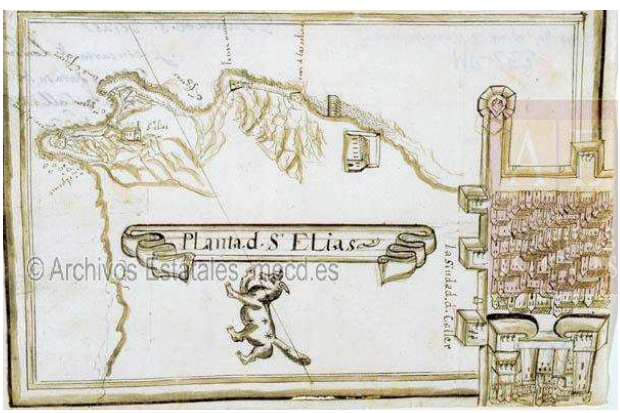

Fig. 2. Planta d[e] S[ant]t Elias, 1623 (foto tratta da Scanu, Podda, Scanu, 2017, p. 999).

La zona di Sant'Elia fu inserita dal Vivas in un progetto complessivo di difesa dell'intera area costiera del Golfo di Cagliari e illustrata in una carta che mostra un'ampia porzione dell'entroterra e del litorale tra Capo Pula, Cagliari e Quartu (Fig. 3). Il disegno ci è pervenuto in più copie, lievemente differenti tra loro, allegate a corredo di alcune relazioni sullo stato delle difese trasmesse dal viceré al sovrano nel corso del 1625 (Nocco, 2016 e 2018).

La città di Cagliari, identificata con il perimetro fortificato di Castello e Marina, figura al centro del disegno. Poco distante e rappresentato come borgo a sé, è Stampace, disegnato con una piccola veduta prospettica. Piccole vedute illustrano anche Quartu, Pauli, Pirri, il castello di San Michele, le chiese di Bonaria e San Bartolomeo e le dieci torri costiere del Cagliaritano (Principe, 1981 e 1982).

Una copia della carta (pubblicata per la prima volta da Scanu, Podda, Scanu, 2017, da cui è tratta la Fig. 3), a differenza delle altre versioni (Principe, 1981, fig. 44; 1982, p. 195), riportava nella parte inferiore del foglio una legenda corrispondente ad altrettanti numeri e parti del disegno, che fanno riferimento ai luoghi nei pressi di Cagliari in cui sarebbe potuto sbarcare il nemico 
e alle opere di difesa da realizzarvi. Sono segnalate l'area tra il bastione di Sant'Agostino e la Scafa, a presidio dei quali stanno le torri de la Escafa e de La Guardiola; ${ }^{6}$ l'area delle saline; la plazuela tra la torre de Las Salinas e Cala de Moscas, in cui compare la T. El Salvador. Quest'ultima era segnalata come La Torre Nue$v a$ nella carta di Sant'Elia (Fig. 2) di due anni prima.

Tre fuertecillos sono suggeriti invece a controllo degli stagni: il primo antistante la spiaggia del Pouet, per controllare l'estaño de las salinas, insieme a una torre (senza nome, ma dovrebbe essere quella detta dal Fara "Quarti seu ostii fluminis"); un altro forte si trova tra questo stagno e quello di san Julian, il terzo tra quest'ultimo e l'estaño de Pauli. Altro punto strategico segnalato è il castello di San Michele che "asigura los socorsos de la isla para Caller".

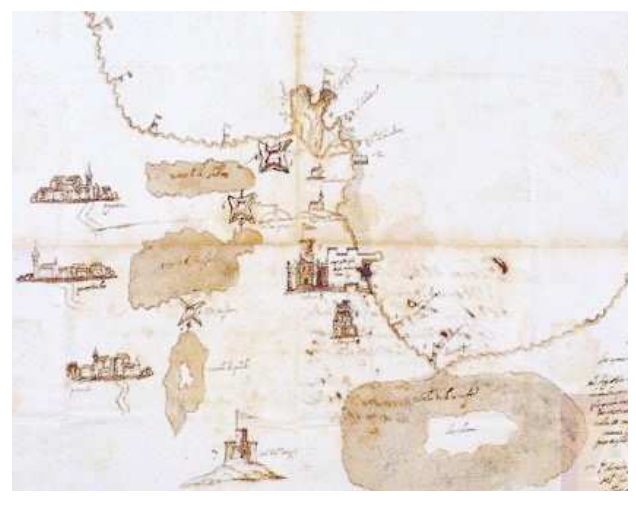

Fig. 3. Dettaglio della Planta del castillo de Càller y reparos y fortificaes. que el Virrey hazía pa. deffender los desembarcaderos en caso q. enemigos intentasen algo, 1625 (da Scanu, Podda, Scanu, 2017, p. 1001).

Il progetto articolato del Vivas non poté essere attuato se non in minima parte e con un certo ritardo per la cronica mancanza di fondi che assillava le casse regie. Il viceré, infatti, riuscì a mala pena ad avviare il cantiere per la torre prevista nei pressi di Calamosca, ma i lavori furono presto interrotti.

Tuttavia, nel 1637 l'enorme impressione suscitata dall'invasione francese di Oristano giunse fino a Cagliari e in tale circostanza si provvide non solo a rinforzare le mura del settore orientale del Castello e della Marina (Nocco, 2016), ma fu anche l'occasione per riprendere, a distanza di dieci anni, i lavori per la torre di Calamosca progettata dal Vivas, a conferma dell'utilità del presidio (Mele, 2000).

\section{Conclusioni}

Dopo questo rapido excursus, si rivela quanto mai interessante il confronto tra le carte disegnate all'epoca del viceré Vivas (soprattutto quella appena descritta) e quella disegnata nel secolo precedente da Rocco Capellino, entrambe aventi per oggetto la parte del Golfo di Cagliari compresa tra Capo Pula e il litorale di Quartu, la città di Cagliari e l'entroterra con i centri abitati presenti, peraltro ritratte dallo stesso punto di osservazione posto sul versante occidentale.

Le carte di cui si è parlato in queste pagine appaiono fortemente connotate dal loro status di documenti dal preminente valore militare finalizzati alla difesa. Questa destinazione ha fatto in modo che fossero gelosamente custodite negli archivi segreti del re, alcune -come abbiamo visto- sono rimaste inedite fino a pochi anni orsono e talvolta i loro autori sono ancora da identificare, un fatto frequente per gli ingegneri del XVII secolo. Meno conosciuti, ma non per questo meno importanti, molto resta ancora da indagare su queste figure professionali, sul loro lavoro, sulle opere realizzate, nonché sul ruolo di eventuali assistenti -disegnatori e pittori- a supporto dell'attività degli ingegneri, come già accadeva nel secolo precedente (Cámara, 2005).

Tuttavia, in questi disegni, sebbene nati come strumento per il governo del territorio, si osserva il ricorso ad una tecnica di rappresentazione mista, con elementi pittorici e prospettici uniti a raffigurazioni in piano, scelta che conferisce una certa gradevolezza alle immagini.

Le carte prodotte sotto il viceré Vivas, infine, redatte a distanza di circa mezzo secolo da quelle di Rocco Capellino, offrono uno spaccato che consente in alcuni casi di analizzare complessivamente il territorio nel Cagliaritano, visto quale "sistema fortificato", rivelando dati interessanti sulla riorganizzazione del sistema difensivo dell'isola promosso dai viceré, sugli interventi compiuti, o talvolta solo progettati, suggerendo 
vari spunti di riflessione che meritano ulteriori indagini.

\section{Note}

Ricerca svolta nell'ambito del progetto Città tra mare e laguna: da Santa Gilla a Cagliari. Aspetti archeologici, geologici, storici, insediativi e sociali, P.I. M. Verga (ISEM-CNR), con fondi L.R. 7/2007 Regione Sardegna.

${ }^{1}$ La documentazione offre, seppur di rado, puntuali riferimenti a immagini dell'isola prodotte tra XVI e XVII secolo finora non rinvenute negli archivi o identificate con certezza. Ne sono un esempio la carta del pittore genovese Ferra o Ferro (la cui identificazione con un affresco presente a Cagliari attende ancora conferme), voluta dal vicerè Juan Coloma al pari di quella disegnata dal pittore Raxis a corredo della relazione di Marco Antonio Camós, incaricato nel 1572 di individuare i siti in cui edificare le torri costiere. Su quest'ultima vicenda si vedano in particolare gli studi di Zedda Macció (2007, 2008), la quale ha identificato presso l'Archivio Generale di Simancas una carta anonima e non datata della Sardegna che sarebbe riconducibile all'attività del Camós e che, sebbene non fosse quella allegata alla relazione ufficiale, costituisce comunque "la prima e per ora unica immagine della Sardegna moderna [...] sicuramente promossa dalle istituzioni spagnole" (Ivi, p. 336). Risulta ancora irreperibile, invece, la "planta del Reyno, la mejor que e podido haver", che il viceré Vivas inviò al sovrano nel 1625 insieme a una relazione e ad altre carte topografiche, tuttora conservate (Nocco, 2016).

2 Poche e frammentarie sono le notizie sull'ingegnere cremonese, i cui elaborati sono conservati nella Biblioteca Apostolica Vaticana
(Codice Barberini Latino 4414). Si tratta di una relazione del maggio 1577 (in tre redazioni), un disegno della Sardegna (anch'esso in tre diverse redazioni che hanno avuto un ruolo determinante nell'evoluzione della cartografia dell'isola), alcune carte topografiche e piante di piazzeforti. Sull'attività di Capellino si vedano Viganó, 2004; Zedda Macció, 2008 e bibliografia ivi citata.

${ }^{3}$ Tra gli obiettivi del progetto Città tra mare $e$ laguna: da Santa Gilla a Cagliari è anche la ricostruzione della fisionomia e della posizione della cittadella giudicale di Santa Gilla. Sul contenuto dei disegni di Capellino e l'analisi della toponomastica si rinvia a Alberti, 1970.

${ }^{4}$ Così, mentre nel 1575 Juan Çanoguera verificava lo stato delle difese isolane e in particolare delle piazzeforti di Cagliari e Alghero, tre anni dopo il viceré de Moncada predisponeva un piano di difesa globale per garantire a lungo termine la sicurezza dei litorali. In accordo con il sovrano decise di approntare una milizia che intervenisse su segnalazione di un circuito di torri d'avvistamento simile a quello costruito nei regni di Valenza, Napoli e Sicilia, affidando a città e baroni la fortificazione delle località che erano soliti far presidiare da gruppi di sentinelle armate e lasciando i rimanenti tratti di litorale all'Amministrazione delle torri (Mele, 2000).

${ }^{5}$ Sul lato destro della carta spicca una singolare rappresentazione della città di Cagliari e della sua trama urbana, per la cui analisi si rinvia a Nocco, 2016 e 2018.

${ }^{6}$ Queste due torri erano a carico della città di Cagliari, che nel Parlamento del 1614 aveva chiesto che potessero essere mantenute dall'Amministrazione del reale, non incontrando però il favore del sovrano (Ortu, 1995).

\section{Bibliography}

Alberti, O. (1970). "Le carte della Sardegna di Rocco Capellino", Nuovo Bollettino Bibliografico Sardo, 70, pp. 3-9; 71. pp. 3-10; 72. pp. 3-7.

Cámara, A. (1998). Fortificación y ciudad en los reinos de Felipe II. Nerea Ed., Madrid.

Cámara, A. coord. (2005). Los ingenieros militares de la Monarquía hispánica en los siglos XVII y XVIII, Ministerio de Defensa Ed., Madrid. 
Dai Prà, E. (2010). "Il paesaggio liminare nella cartografia storica tra metafora e progetto", Bollettino dell'Associazione Italiana di Cartografia, 139-140, pp. 167-179.

Gambi, L. (1987). "Considerazioni a chiusura", in Cartografia e Istituzioni in età moderna, 27, 2, pp. 847-858, 855.

Manconi, F. (2001). "Le istruzioni di Carlo V al viceré Cardona per il governo della Sardegna (1534)", in Dal mondo antico all'età contemporanea. Studi in onore di Manlio Brigaglia offerti dal Dipartimento di Storia dell'Università di Sassari, Carocci Ed., Roma, pp. 373-395.

Mele, G. (2000). Torri e cannoni. La difesa costiera in Sardegna nell'età moderna, Sassari Ed., Sardinia.

Mele, G. (2006). Documenti sulla difesa militare della Sardegna in età spagnola, Fondazione Banco di Sardegna Ed., Sassari.

Mele, G. (2017). La rivoluzione militare in Sardegna: fortificazioni, presidi e milizia territoriale. Fonti d'archivio (1553-1611), Universitas Studiorum Ed., Mantova.

Nocco, S. (2016). "Cagliari nel Seicento. Forma e rappresentazione di una piazzaforte", in Defensive architecture of the Mediterranean XV to XVIII Centuries. Proceedings of the International Conference on Modern Age Fortifications of the Mediterranean Coast. FORTMED 2016, G. Verdiani Ed., Firenze, vol. III, pp. 185-192.

Nocco, S. (2018). “Cagliari nella cartografia storica: la realtà in evoluzione di una capitale”. in Guia Marin, L.J. coord., Centri di potere nel Mediterraneo occidentale. Dal Medioevo alla fine dell'Antico Regime, Franco Angeli Ed., Milano, pp. 121-130.

Ortu, G.G. (1995). Il Parlamento del viceré Carlo de Borja, duca di Gandia (1614). Consiglio Regionale della Sardegna Ed., Cagliari.

Principe, I. (1981). Cagliari. Laterza Ed., Roma-Bari.

Principe, I. (1982). "La Sardegna spagnola: cristallizzazione di una società periferica", in Il progetto del disegno. Città e territori italiani nell'Archivo General di Simancas, Casa del Libro Ed., Reggio Calabria/Roma, pp. 189-199.

Quaini, M. (1974). "Catalogna e Liguria nella cartografia nautica e nei portolani medievali”, in Atti del I Congresso storico (Liguria-Catalogna, ecc. 14-19 ottobre 1969), Istituto internazionale di studi liguri Ed., Bordighera, pp. $550-571$.

Rassu, M. (2005). Sentinelle del mare. Le torri della difesa costiera della Sardegna. Grafiche del Parteolla Ed., Dolianova.

Scanu, G; Podda, C; Scanu, G. (2017). "Osservazioni preliminari su alcune mappe della Sardegna spagnola", in Atti 21 Conferenza Nazionale Asita ed Expo 2017, (Online Ed.), pp. 987-1006.

Urban, M.B. (2000). Cagliari aragonese. Topografia e insediamento, CNR-IRII Ed., Cagliari.

Viganó, M. (2004). «El fratin mi ynginiero». I Paleari Fratino da Morcote ingegneri militari ticinesi in Spagna (XVI-XVII secolo), Casagrande Ed., Bellinzona.

Zedda Macció, I. (2007). "Insularità e rappresentazione dello spazio costiero. Frontiere del Regnum Sardiniae nell'Età Moderna", in Conti, S. coord., Amate sponde. Le rappresentazioni dei paesaggi costieri mediterranei, Grafica Art Ed., Formia, pp. 313-355.

Zedda Macció, I. (2008). "Cartografie e difesa nella Sardegna del Cinquecento. Pratiche geografiche, carte segrete e immagini pubbliche", in "Contra Moros y Turcos». Politiche e sistemi di difesa degli Stati mediterranei della Corona di Spagna in Età Moderna, CNR-ISEM Ed., Cagliari, pp. 633-684. 